\title{
REFLEXIÓN DE LA ENSEÑANZA-APRENDIZAJE DEL DERECHO EN EL SIGLO XXI Y EL MODELO BASADO EN COMPETENCIAS
}

\author{
Paula Comellas Angulo \\ Universidad Católica de Colombia
}

\section{Resumen}

El objetivo principal de este artículo es hacer una reflexión, en ningún caso derivada de la investigación, en torno a la influencia que ejercen la globalización e internacionalización en la enseñanza-aprendizaje del Derecho en el siglo XXI. Con base en la convergencia global en materia educativa y en el modelo imperante del aprendizaje basado en competencias, se analizarán los nuevos perfiles académico-profesionales del jurista de nuestro siglo y las competencias que tendrían que regir la enseñanza-aprendizaje del Derecho en la sociedad actual. Además, se hará un análisis de la presente sociedad y del cambio del paradigma en la enseñanza-aprendizaje.

Palabras clave: Derecho, gestión educativa, método de aprendizaje, abogado

La autora: abogada, especialista en Currículo y Pedagogía, de la Universidad de los Andes, magíster en Investigación en Ciencias Jurídicas, doctoranda en Derecho Internacional, de la Universidad de Deusto, Bilbao (España). Docente de cátedra en la Facultad de Derecho de la Universidad de los Andes, docente e investigadora de la Facultad de Derecho de la Universidad Católica de Colombia.

Correo electrónico: paulacomellas@hotmail.com

Recibido: 7 de mayo de 2015; evaluado: 29 de mayo de 2015; aceptado: 1 de junio de 2015.

NOVUM JUS • ISSN: 1692-6013 • Volumen 9 No .1 • Enero - Junio 2015 • Págs. 81-102 


\title{
A REFLECTION ON THE TEACHING-LEARNING PROCESSES IN LAW IN XXI CENTURY AND THE COMPETENCY BASED MODEL
}

\author{
Paula Comellas Angulo \\ Universidad Católica de Colombia
}

\begin{abstract}
The purpose of this paper is to present a reflection-in no way derived from research —upon the influence of globalization and internationalization in the teaching-learning of Law in the XXI Century. Based on the global convergence in education and the prevailing model of Competency Based Learning, we analyze the new academic-professional profiles of the jurists of the new century and the competencies that will govern the teaching-learning of law in modern society. We also analyze the present society and the change that has taken place in the teaching-learning paradigm.
\end{abstract}

Key words: Law, educational management, learning method, lawyer

About the author: Lawyer, MA in Legal Sciences and doctoral candidate for International Law (Universidad de Deusto, Bilbao, Spain). She has a specialization in Curricula and Pedagogy (CIFE, Universidad de los Andes). Part-time teacher at the Law School at Universidad de los Andes, and is a teacher and researcher at Universidad Católica de Colombia.Email: paulacomellas@hotmail.com

Received: May 7, 2015; reviewed: May 29, 2015; accepted: June 1, 2015. 
Reflexión de la enseñanza-aprendizaje del derecho en el siglo XXI...

\section{Introducción}

La globalización, la internacionalización y el desarrollo de las nuevas tecnologías, entre otros factores, han provocado transformaciones sin precedentes en amplios sectores de la sociedad actual. Este hecho ha influido de una manera sin par en la educación superior, la cual se encuentra inmersa en un nuevo paradigma entre la convergencia global educativa y la definición de nuevos perfiles académico-profesionales.

La universidad, como institución al servicio de la sociedad, debe adaptarse a estos cambios, que se ven reflejados sobre todo en la educación transnacional y en la movilidad de estudiantes, para evitar formarlos en competencias desfasadas de las necesidades de la sociedad actual. De acuerdo con lo anterior, es necesario definir nuevos perfiles académico-profesionales y crear puentes entre la sociedad y la universidad. El modelo de aprendizaje basado en competencias ha sido elegido como el más adecuado para llevar a cabo este cambio de paradigma en la formación de los profesionales del siglo XXI.

El Derecho no ha quedado al margen de estas transformaciones, ya que cada día son más los negocios jurídicos que se caracterizan por poseer un elemento extranjero. El incremento de estas transacciones hace que las situaciones privadas internacionales predominen cada día más en esta área, lo que debe conducir también a generar cambios en la manera como se forma a los juristas globales. Asimismo, las organizaciones internacionales desempeñan un rol muy destacado en la creación e interpretación del Derecho, lo que hace que seamos testigos de la aparición de nuevas instituciones jurídicas y, a su vez, de la reformulación de las tradicionales.

El objetivo nuclear de este artículo es analizar los retos que se enfrenta la enseñanzaaprendizaje del Derecho en el siglo XXI y reflexionar en torno al modo de garantizar la no desarticulación entre las competencias que el estudiante de Derecho adquiere a lo largo de la carrera y las que demanda el mercado laboral.

\section{La universidad en la sociedad del siglo XXI}

\subsection{Análisis de la sociedad actual}

La sociedad del siglo XXI se caracteriza por la globalización, internacionalización y transnacionalización de todas sus esferas. Respecto a la primera, el término globali-

NOVUM JUS • ISSN: 1692-6013 • Volumen 9 No 1 • Enero - Junio 2015 • Págs. 81-102 
zación surgió en relación con la dimensión transfronteriza que ha predominado en las últimas décadas en las relaciones económicas, políticas, sociales y culturales. ${ }^{1}$ La doctrina señala como factores originarios de este fenómeno la privatización de las empresas y, sobre todo, la apertura de los mercados nacionales mediante los tratados de libre comercio. ${ }^{2}$

El concepto de Derecho Transnacional se atribuye a Philip C. Jessup Storrs, a raíz de que comenzara a utilizar el término en sus clases magistrales en la Universidad de Yale para referirse al "Derecho que regula acciones o eventos que trascienden las fronteras nacionales". ${ }^{3}$

Los rasgos enunciados y el desarrollo de la tecnología de la información y de la comunicación (TIC) a finales de la década del noventa dieron lugar a la llamada "nueva economía", en alusión a la influencia que estos dos fenómenos ejercen en la sociedad. ${ }^{4}$ En este contexto surgió el término "sociedad de la información" que, en términos sencillos, se refiere a un escenario de acceso y transmisión de información más ágil y eficiente debido a Internet. Puesto que era necesario administrar y seleccionar la vasta cantidad de información a la que se tenía acceso, la doctrina comenzó a acuñar el término "sociedad del conocimiento". Lo que distingue a esta respecto a aquella es que en la sociedad del conocimiento los individuos son capaces de seleccionar la información que les es útil y, del mismo modo, desechar la que no es fiel a sus intereses.

El Derecho no puede quedar al margen de los anteriores cambios. En la actualidad han proliferado numerosos actores de la sociedad civil organizada que desempeñan un papel destacado en la creación del Derecho. ${ }^{5}$ Ante esta realidad, si la forma en la

1 Ulrich Beck. ¿Qué es la globalización? Falacias del globalismo, respuestas a la globalización (Barcelona: Paidós, 1998), 56

2 Para una visión más profunda, puede verse XXII Congreso Mundial de Filosofía Jurídica y Social, "Declaración de Granada sobre la globalización", http://www.globalizacion.org/globalizacion/GlbzDeclaracionGranada. htm (acceso febrero 4, 2013).

3 Peer Zumbansen, "Transnational Law", en Elgar Encyclopedia of Comparative Law, (Londres: Edward Elgar Publishing Ltd., 2006), 738.

$4 \quad$ En el siglo XXI, la sociedad está implicada en una gran cantidad de transformaciones y cambios constantes producidos por diversos factores, como las nuevas tecnologías, el mercado global, la gestión del conocimiento, etc., conocidos con el nombre de la "nueva economía". Antonio Sáenz de Miera, La universidad en la nueva economía (Madrid: Secretaría General Técnica, 2002), 9.

5 Señala De Julios Campuzano: "El nuevo paradigma que la globalización inaugura nos presenta un derecho dependiente del subsistema económico, al cual se subordina un derecho versátil, adaptable y flexible que poco o nada tiene que ver con esa apariencia de cientificidad del sistema jurídico completo, unitario y coherente en la modernidad". Alfonso de Julios Campuzano, La transición paradigmática de la teoría jurídica. El Derecho ante la globalización (Madrid: Dykinson, 2009), 77. 
Reflexión de la enseñanza-aprendizaje del derecho en el siglo XXI...

que se practica y se crea el Derecho ha evolucionado, de igual manera tendría que evolucionar el perfil académico-profesional del jurista del siglo XXI y dar cabida a los cambios necesarios en los métodos de enseñanza-aprendizaje. ${ }^{6}$

\subsection{Reformas educativas globales y el proyecto Tuning Educational Structures}

Quizás con la intención de enfrentar los retos enunciados, la educación superior global sufrió un punto de inflexión, tras el establecimiento del Espacio Europeo de Educación Superior (en adelante, EEES). La raíz de este cambio se encuentra en la Magna Charta Universitatum, ${ }^{7}$ documento que nació como fruto de una reunión celebrada para conmemorar el aniversario novecientos de la Universidad de Bolonia, ${ }^{8}$ cuyo objetivo principal era analizar el papel de las universidades a la luz de "una sociedad que se transforma y se internacionaliza". 9

La Magna Charta Universitatum supuso un punto de partida en la reclamación de las transformaciones que debían darse en la educación superior para adaptarse a la sociedad global. Así, a esta Carta le siguió la Declaración de la Sorbona, que

En este mismo sentido, Litvinoff señala: "Cualquiera que sea su especialización, la actividad profesional de los abogados que tienen a su cargo estas transacciones — las cuales deben contener obligatoriamente una ley aplicable, un foro y una cláusula compromisoria, así como la influencia que tiene cada vez más el aumento de la información sobre laudos de prestigiosos tribunales de arbitramiento internacional— está en proceso de desarrollar una nueva Lex Mercatoria, es decir, un conjunto de reglas prácticas orientadas más hacia los resultados prácticos aceptables, que a la reafirmación de principios establecidos". Saul Litvinoff, "Derecho Global, Derecho Civil y Common Law", Foro de Derecho Mercantil: Revista Internacional, núm. 3 (abril-junio 2004), 102

6 Autores que apuntan en esta dirección: José Ramiro Viso Alonso, Qué son las competencias. Enseñar y aprender por competencias (Madrid: EOS, 2010), 13; Alfredo Jiménez Eguizábal (dir.), Proyecto Piloto de adaptación de asignaturas de Pedagogía y Psicología al sistema de créditos europeos ECTS (Burgos: Universidad de Burgos, 2005), 11.

7 En la actualidad, la Magna Charta Universitatum ha sido firmada por 721 universidades correspondientes a los siguientes 79 países: Albania, Alemania, Andorra, Argentina, Armenia, Australia, Austria, Azerbaiyán, Bahréin, Bélgica, Bosnia-Herzegovina, Brasil, Bulgaria, Camboya, Canadá, Chile, China, Chipre, Colombia, Croacia, Dinamarca, Ecuador, Egipto, El Salvador, Eslovaquia, Eslovenia, España, Estados Unidos, Estonia, Filipinas, Finlandia, Francia, Georgia, Ghana, Grecia, Guatemala, Holanda, Hungría, India, Indonesia, Irán, Irlanda, Islandia, Israel, Italia, Japón, Kazakstán, Kosovo, Kirguistán, Letonia, Líbano, Lituania, Macedonia, Malta, Marruecos, México, Moldavia, Nigeria, Noruega, Paraguay, Perú, Polonia, Portugal, Puerto Rico, Reino Unido, República Checa, República Dominicana, Rumania, Rusia, Serbia, Sudáfrica, Suecia, Suiza, Túnez, Turkmenistán, Turquía, Ucrania, Uzbekistán, Venezuela y Zimbabue. Magna Charta Universitatum (Bolonia, 18 de septiembre de 1988).

8 En concreto, se reunieron los rectores de las universidades de Bolonia, París I, Lovaina, Utrecht y Barcelona.

9 Debe ser tenido en cuenta el contexto en el que fue redactada la Magna Charta Universitatum, pues cuatro años después de su redacción estaba prevista la supresión definitiva de las fronteras intercomunitarias y se preveía una colaboración en aumento entre los Estados europeos. Magna Charta Universitatum, Preámbulo.

NOVUM JUS • ISSN: 1692-6013 • Volumen 9 Nº. 1 • Enero - Junio 2015 • Págs. 81-102 
fue firmada en París en 1998. ${ }^{10}$ Esta última consagró los principios de la Magna Charta Universitatum y su impacto fue notable, ya que un año después se firmó la Declaración de Bolonia, que estableció el EEES y la Europa del conocimiento, cuya implementación en todas las universidades firmantes estaba prevista para el año 2010. ${ }^{11}$

La Declaración de Bolonia fue el comienzo de la convergencia europea en materia educativa. Su objetivo principal era la armonización de los títulos superiores de educación con el fin de facilitar la movilidad de estudiantes y trabajadores en el seno del continente europeo. ${ }^{12}$ Con esta Declaración también se pretendía adaptar la educación a los cambios que se habían producido en la sociedad, en concreto, con el desarrollo de las nuevas tecnologías y la sociedad del conocimiento, en busca de la formación de individuos en las competencias necesarias para desenvolverse en este nuevo escenario. ${ }^{13}$

Para ello, se estableció un sistema educativo superior de tres ciclos — grado, posgrado y doctorado—, la implementación de nuevas metodologías de enseñanza-aprendizaje y un nuevo sistema de medición del trabajo de los estudiantes mediante el Sistema Europeo de Transferencia de Créditos (ECTS, por su nombre en inglés).

10 En este caso, el motivo de la reunión fue el aniversario setecientos de la Universidad de la Sorbona y en ella estuvieron presentes los ministros de Educación de Francia, Italia, Alemania e Inglaterra. En esta Declaración se plasman las ideas surgidas en la anterior reunión de Bolonia y de nuevo se hace hincapié en las transformaciones que están sufriendo tanto la sociedad como el mercado laboral y la consecuente necesidad de una reforma educativa ligada a dichos cambios. Ministros europeos de Educación Superior, Declaración de La Sorbona (La Sorbona, París, 25 de mayo de 1998).

11 "Por la presente Declaración, hacemos público nuestro compromiso en favor de estos objetivos —en el marco de nuestras competencias institucionales y en el pleno respeto de la diversidad de las culturas, de las lenguas, los sistemas nacionales de enseñanza y la autonomía universitaria—, con el fin de consolidar el espacio europeo de enseñanza superior". Ministros europeos de Educación Superior, Declaración de Bolonia, 3. Cabe destacar que esta Declaración fue ratificada en 1999 por 29 países y en la actualidad 45 Estados forman parte del EEES.

12 Este proceso de convergencia europeo está basado en seis puntos: el Sistema Europeo de Transferencia de Créditos (European Credit Transfer System, ECTS); el suplemento europeo al título; un sistema de titulaciones de dos ciclos (grado y máster); la promoción de la movilidad tanto de los estudiantes como de profesores y PAS; el impulso de la cooperación europea en materia de aseguramiento de la calidad con miras al desarrollo de criterios y metodologías comparables; por último, el aumento de la dimensión europea de la enseñanza superior, en especial respecto a la elaboración de programas de estudios, de cooperación interinstitucional, de movilidad y los integrados de estudios, formación e investigación. Ministros europeos de Educación Superior, Declaración de Bolonia, 2-3.

13 "La Europa de los conocimientos está ampliamente reconocida como factor insustituible para el crecimiento social humano, y como elemento indispensable para consolidar y enriquecer la ciudadanía europea, confiriendo a sus ciudadanos las competencias necesarias para afrontar los retos del nuevo milenio, junto con la concienciación de los valores compartidos y de la pertenencia a un espacio social y cultural común". Ministros europeos de Educación Superior, Declaración de Bolonia, 1. 
Reflexión de la enseñanza-aprendizaje del derecho en el siglo XXI...

Con el fin de ofrecer una propuesta metodológica práctica que lograra una verdadera implementación de los objetivos plasmados en el EEES, nació en el año 2000 el proyecto Tuning Educational Structures in Europe (en adelante, Tuning). Este proyecto, respaldado por la Comisión Europea, fue ideado por Julia González (Universidad de Deusto) y Robert Wagenaar (Universidad de Groningen, Holanda). Su lema es la armonización de las estructuras y los programas educativos con respeto por la diversidad y autonomía de cada sistema. ${ }^{14}$ Su objetivo es definir puntos de referencia, de convergencia y de entendimiento mutuo en aras de la correcta implementación de los propósitos perseguidos por la Declaración de Bolonia.

Este proyecto tuvo un papel clave en la definición de las competencias tanto genéricas como específicas para más de treinta áreas de estudio —entre ellas, el Derecho- ${ }^{15}$ y en la metodología para lograr que los estudiantes alcanzaran dichas competencias.

El proyecto está asentado sobre cinco pilares: las competencias genéricas, las competencias específicas, la función de los ECTS como sistema de acumulación (no como sistema de transferencia), los enfoques de aprendizaje, enseñanza, evaluación y la promoción de la calidad en el proceso educativo. ${ }^{16}$ Lo más destacado de la metodología es que, para la definición de las competencias de cada perfil académico-profesional, se parte de las necesidades sociales de cada titulación por medio de la aplicación de encuestas a empleadores, académicos, graduados y estudiantes. Los datos arrojados por estas encuestas se traducen en resultados del aprendizaje y, por ende, en competencias genéricas y específicas a desarrollar por los estudiantes. Lo anterior se incluye en el currículo universitario, con un enfoque claro de los métodos de enseñanza-aprendizaje y evaluación acordes con el modelo basado en competencias.

Sin lugar a dudas, el desarrollo de programas de estudios comparables, compatibles y transparentes es uno de los requisitos sine qua non para hallar la ansiada convergencia en materia educativa. Por otra parte, elaborar los títulos con base en los resultados del aprendizaje y de las competencias contribuye a una enseñanza

14 Para una visión más profunda del proyecto puede verse Julia González y Robert Wagennar, Tuning Educational Structures in Europe. La contribución de las universidades al proceso de Bolonia. Una introducción (Bilbao: Universidad de Deusto, 2006).

15 Agricultura, Arquitectura, Historia del Arte, Administración de Empresas, Química, Ingeniería Civil, Informática, Danza, Diseño, Ciencias Sociales, Economía, Educación, Estudios Europeos, Bellas Artes, Estudios de Género, Geografía, Historia, Relaciones Internacionales, Derecho, Literatura, Lingüística, Medicina, Matemáticas, Música, Enfermería, Terapia Ocupacional, Psicología, Fisioterapia, Ciencias Políticas, Radiología, Trabajo Social, Teatro y Teología.

16 González y Wagennar, Tuning Educational Structures in Europe, 150.

NOVUM JUS • ISSN: 1692-6013 • Volumen 9 Nº. 1 • Enero - Junio 2015 • Págs. 81-102 
centrada en el aprendizaje del estudiante, lo que supone un cambio en el rol tradicional del profesor.

A pesar de que esta reforma educativa en educación superior se produjo en el continente europeo, tuvo repercusión global. Esto se evidencia, sin ir más lejos, en el proyecto Tuning que, aunque fue creado con una visión europea, se está implementado en América Latina, Rusia, Estados Unidos y África y será lanzado en más países. Este fenómeno responde al cambio de paradigma de la educación superior.

\section{Cambio de paradigma en la enseñanza-aprendizaje del Derecho}

\subsection{De la enseñanza al aprendizaje del Derecho}

Con esta reforma educativa, no solo se pretendía una reestructuración de los estudios tendiente a la armonización de titulaciones, sino también un cambio metodológico de la enseñanza-aprendizaje del Derecho y del rol tradicional del estudiante y del profesor. En este nuevo paradigma, se ansía que todos los docentes centren sus esfuerzos en lo que los estudiantes aprenden y no tanto en lo que ellos enseñan. ${ }^{17}$ La idea es que el profesor se convierta en un guía del proceso de aprendizaje del alumno. La lección magistral no desaparece, pues es evidente que los conocimientos teóricos son fundamentales, pero se intercalan con los desempeños activos de los estudiantes, como resolución de ejercicios prácticos, el aprendizaje basado en problemas, el desarrollo de debates jurídicos o la exposición de trabajos grupales.

El hecho de que con la instauración del EEES se pretenda un papel más activo del alumno en la formación de su propio aprendizaje supone que las competencias docentes también varíen, con el fin de adaptarse a esta nueva situación. Así, el perfil del profesor universitario tendrá que incluir otro tipo de competencias docentes que van más allá del conocimiento teórico-práctico de la asignatura, por ejemplo, capacidad para el diseño de tareas de estudio y aprendizaje autónomo, criterios de evaluación y relación entre alumno y profesor.

\footnotetext{
17 "Puede parecer una obviedad decir que los profesores están para que los alumnos aprendan, pero si tanto se habla ahora de la enseñanza centrada en el alumno, alguna razón habrá. Quizás porque, casi sin darnos cuenta, los profesores hemos estado prestando más atención a lo que tenemos que hacer nosotros para enseñar (como es preparar clases, explicar, examinar, calificar) que a lo que tienen que hacer los alumnos para aprender". Pedro Morales Vallejo, "Implicaciones para el profesor de una enseñanza centrada en el alumno", Miscelánea Comillas, 64 núm. 124 (2006): 11-12.
} 
Reflexión de la enseñanza-aprendizaje del derecho en el siglo XXI...

En última instancia, la clave de este nuevo paradigma reside en la reflexión necesaria de todo profesor sobre lo que espera que sus estudiantes aprendan a lo largo del curso, la metodología que va a emplear para propiciar ese aprendizaje y el sistema de evaluación que sería acorde para alinear estos tres factores.

\subsection{Nuevos perfiles académico-profesionales: el jurista global del siglo XXI}

Es necesario redefinir el perfil académico-profesional del jurista global y determinar la clasificación de competencias genéricas y específicas que conforman dicho perfil. ${ }^{18}$

Sin lugar a dudas, este perfil académico-profesional debe atender a las necesidades de la sociedad actual. Asimismo, hay que tener presentes las necesidades futuras, como establece el informe New Skills for New Jobs, elaborado en 2010 por un grupo de expertos de la Comisión Europea. Este Informe concluye que en las próximas décadas seremos testigos de la aparición de nuevas profesiones, a consecuencia de los cambios acaecidos en la sociedad del siglo XXI, ${ }^{19}$ por lo que su objetivo nuclear es alertar de sobre la aparición de estas nuevas profesiones con el fin de formar a los estudiantes en las competencias que les serán requeridas en el mercado laboral.

Respecto al perfil profesional del jurista global, los cambios producidos en esta área responden al aumento de las transacciones internacionales, ya que cada día son más los negocios jurídicos que tienen como característica principal un elemento extranjero. Asimismo, los efectos jurídicos de la globalización y la proliferación de distintas organizaciones internacionales han modificado la manera de crear y practicar el Derecho. ${ }^{20}$

Antes de analizar el aprendizaje basado en competencias, es preciso tener en cuenta que el concepto de competencia estaba reservado al ámbito laboral, en alusión al conjunto de habilidades profesionales que todo aspirante debía poseer para determinado puesto. No obstante, con la Declaración de Bolonia y la consiguiente convergencia global en materia educativa, comenzó a acuñarse el término en el

\footnotetext{
18 Jaime Rodríguez-Arana y Rafael Palomino Lozano (dirs.), Enseñar Derecho en el Siglo XXI. Una guía práctica sobre el Grado en Derecho (Pamplona: Thomson Aranzadi, 2009), 93.

19 Comisión Europea, "New Skills for New Jobs", http://ec.europa.eu/social/main.jsp?catId=568 (acceso marzo 1, 2013),

20 Aneca, "Libro Blanco. Título de grado en Derecho", http://www.aneca.es/var/media/150240/libroblanco_derecho_def.pdf (acceso marzo 1, 2013).
}

NOVUM JUS • ISSN: 1692-6013 • Volumen 9 No. 1 • Enero - Junio 2015 • Págs. 81-102 
mundo académico, hasta implementarse el modelo del aprendizaje basado en competencias, como será explicado a continuación. ${ }^{21}$

\section{Aprendizaje basado en competencias (ABC)}

\subsection{Contextualización}

El aprendizaje basado en competencias ha sido el modelo elegido por la mayoría de los países para implementar los objetivos consagrados en la Declaración de Bolonia que, como fue expuesto, se ha difundido por todo el mundo. La Declaración de Bolonia expresó que los cambios educativos debían tender a formar estudiantes en "las competencias necesarias para responder a los retos del nuevo milenio y reforzar los valores compartidos y de la pertinencia a un espacio social y cultural común". ${ }^{22}$

En términos generales, este modelo de enseñanza-aprendizaje tiene por objetivo que los estudiantes adquieran una serie de competencias genéricas y específicas acordes con las necesidades de la sociedad del siglo XXI. ${ }^{23}$

El término competencia proviene del latín cum y petere, en referencia a la "capacidad para concurrir, coincidir en la dirección". El proyecto Tuning define las competencias como "lo que una persona es capaz o competente de ejecutar, el grado de preparación, suficiencia y/o responsabilidad para ciertas tareas". ${ }^{24}$

Ahora bien, cabe diferenciar entre las competencias genéricas y las específicas. Las primeras son compartidas por todos los pregrados, mientras las específicas, como su propio nombre indica, corresponden a cada área del conocimiento. Dentro de las competencias es posible realizar una clasificación entre instrumentales, interpersonales y sistémicas.

Las competencias genéricas instrumentales guardan relación con capacidades cognitivas, metodológicas, tecnológicas y lingüísticas. Por su parte, las genéricas

21 "Una condición básica para la construcción de una enseñanza orientada a las competencias es disponer de un perfil laboral y de formación con el que las exigencias que se plantean a los titulados coincidan con las competencias que se requieren en el mercado laboral". Juan Antonio García Fraile y Carmen Saban Vera, Un nuevo modelo de formación para el siglo XXI: la enseñanza basada en competencias (Barcelona: Davinci, 2008), 91.

22 Ministros europeos de Educación Superior, Declaración de Bolonia, 1999.

23 García Fraile y Saban Vera, Un nuevo modelo de formación para el siglo XXI, 91.

24 González y Wagennar, Tuning Educational Structures in Europe, 27-28. 
Reflexión de la enseñanza-aprendizaje del derecho en el siglo XXI...

interpersonales aluden a capacidades individuales como las habilidades sociales e interpersonales. Por último, las genéricas sistémicas tienen un carácter holístico, por ejemplo, la capacidad de emprendimiento, organización o liderazgo. Hay que destacar que es necesario adquirir previamente las competencias de tipo instrumental o interpersonal para desarrollar cualquier clase de competencia sistémica. ${ }^{25}$

Para finalizar con esta contextualización, no pueden obviarse las críticas que ha recibido el aprendizaje basado en competencias, que no deja de ser otro que el modelo fundado en la adquisición de objetivos de las décadas del sesenta y del setenta. Entre estas críticas sobresale que se trata de un método conductista, quizá desfasado frente a las bases que deben preceder el aprendizaje moderno y cuya evaluación es finalista. ${ }^{26}$ No obstante, este modelo fue propulsado por la propia Declaración de Bolonia, la cual exigía formar en las competencias de una sociedad internacionalizada y afirmaba que la adquisición de competencias puede compaginarse con otros modelos de aprendizaje que sean más coherentes con el país o la institución en donde se desarrolle.

\subsection{Clasificación de competencias genéricas y específicas para el grado en Derecho}

A la luz de lo expuesto, es necesario establecer las competencias genéricas y específicas que deberán estimularse en los estudiantes de Derecho a lo largo de su formación universitaria.

Para ello, se tomarán en consideración las competencias descritas en el libro Tuning América Latina, porque es el único que hasta este momento ha desarrollado un proyecto en el cual cuenta la opinión de académicos, profesionales y graduados en la definición de las competencias genéricas y específicas básicas del grado en Derecho. El grupo Tuning Derecho América Latina se constituyó en 2006 por Argentina, Bolivia, Brasil, Chile, Colombia, Ecuador, El Salvador, España, México, Nicaragua, Paraguay, Perú, Uruguay y Venezuela. Los objetivos del estudio estaban

25 Aurelio Villa y Manuel Poblete, Aprendizaje basado en competencias. Una propuesta para la evaluación de las competencias genéricas (Bilbao: Universidad de Deusto, 2007), 29-32.

26 La mayoría de las críticas hacia el modelo de aprendizaje basado en competencias parten de la falta de unanimidad que existe en torno al concepto de competencia, lo que conlleva, según estos críticos, a que sea inviable llegar a un consenso sobre cómo, cuáles y cuántas competencias deberían ser desarrolladas por los estudiantes. En este sentido, pueden verse Rosemary Hipkins, The Nature of the Key Competences. A Background Paper (Nueva Zelanda: Wellignton, 2006); Ronald Barnett, Los límites de la competencia. El conocimiento, la educación superior y la sociedad (Barcelona: Gedisa, 2001).

NOVUM JUS • ISSN: 1692-6013 • Volumen 9 Nº 1 • Enero - Junio 2015 • Págs. 81-102 
centrados en modernizar la enseñanza del Derecho, ajustada a los nuevos tiempos y las realidades; lograr un sistema que cubriera a plenitud las necesidades del fututo profesional y de los empleadores; avanzar en un proceso de internacionalización de la enseñanza del Derecho que permitiera tanto la movilidad de estudiantes como de profesionales y académicos e incrementar la calidad del proceso de enseñanzaaprendizaje por medio de un modelo transparente. ${ }^{27}$

En cuanto a las competencias genéricas, el proyecto arrojó como las más importantes:

- Capacidad para aplicar los conocimientos adquiridos.

- Habilidad de adaptarse a nuevas situaciones.

- Gestión de la información.

- Trabajar de manera autónoma.

- Trabajo en equipo y comunicación oral y escrita.

- Capacidad de organización y planificación.

Al igual que en las dos fases previas del Proyecto Tuning realizadas en Europa, se encuestó a académicos, graduados, estudiantes y empleadores en los trece países mencionados y con la misma metodología que en etapas anteriores. Se preguntó sobre la importancia y realización de las siguientes competencias específicas en el área del Derecho:

1. Conocer, interpretar y aplicar los principios generales del Derecho y del ordenamiento jurídico.

2. Conocer, interpretar y aplicar las normas y los principios del sistema jurídico nacional e internacional en casos concretos.

3. Buscar la justicia y equidad en todas las situaciones en las que interviene.

4. Estar comprometido con los derechos humanos y con el Estado social y democrático de Derecho.

5. Capacidad de ejercer su profesión en equipo con colegas.

6. Habilidad para trabajar en equipos interdisciplinarios como experto en Derecho y contribuir de manera efectiva a sus tareas.

7. Comprender los fenómenos políticos, sociales, económicos, personales y psicológicos, entre otros, y considerarlos en la interpretación y aplicación del Derecho.

27 González y Wagennar, Tuning Educational Structures in Europe, 109-131. 
8. Ser consciente de la dimensión ética de las profesiones jurídicas y de la responsabilidad social del graduado en Derecho y actuar en consecuencia.

9. Capacidad de razonar y argumentar jurídicamente.

10. Habilidad de dialogar y debatir desde una perspectiva jurídica, comprender los distintos puntos de vista y articularlos a efecto de proponer una solución razonable.

11. Considerar la pertinencia del uso de medios alternativos en la solución de conflictos.

12. Conocer una lengua extranjera que permita el desempeño eficiente en el ámbito jurídico (inglés, portugués o español).

13. Capacidad para usar la tecnología necesaria en la búsqueda de la información relevante para el desempeño y la actualización profesional.

14. Habilidad para aplicar criterios de investigación científica en su actividad profesional.

15. Capacidad para aplicar sus conocimientos de forma eficaz en un área determinada de su profesión.

16. Habilidad de enfrentar nuevas situaciones y contribuir a la creación de instituciones y soluciones jurídicas en casos generales y particulares.

17. Capacidad para redactar textos y expresarse oralmente en un lenguaje fluido y técnico, con términos jurídicos precisos y claros.

18. Habilidad para analizar una amplia diversidad de trabajos complejos en relación con el Derecho y sintetizar sus argumentos de forma precisa.

19. Capacidad para tomar decisiones jurídicas razonadas.

20. Comprender y relacionar los fundamentos filosóficos y teóricos del Derecho con su aplicación práctica.

21. Demostrar conciencia crítica en el análisis del ordenamiento jurídico.

22. Habilidad para actuar jurídica y técnicamente en diferentes instancias administrativas o judiciales con la debida utilización de procesos, actos y procedimientos.

23. Capacidad para decidir si las circunstancias de hecho están claras para adoptar una decisión fundada en Derecho.

24. Actuar de manera leal, diligente y transparente en la defensa de intereses de las personas a las que representa. ${ }^{28}$

Las seis competencias de mayor importancia en los cuatro grupos consultados (académicos, graduados, estudiantes y empleadores) fueron las siguientes:

28 Resulta interesante analizar también el rango de competencias establecidos en OCDE, "Definition and Selection of Competencies: Theoretical and Conceptual Foundations", http://www.oecd.org/edu/skillsbeyond-school/41529556.pdf (acceso marzo 1, 2013).

NOVUM JUS • ISSN: 1692-6013 • Volumen 9 Nº. 1 • Enero - Junio 2015 • Págs. 81-102 
- Capacidad de razonar y argumentar jurídicamente.

- Actuar de manera leal, diligente y transparente en la defensa de intereses de las personas a las que representa.

- Conocer, interpretar y aplicar los principios generales del Derecho y del ordenamiento jurídico.

- Ser consciente de la dimensión ética de las profesiones jurídicas y de la responsabilidad social del graduado en Derecho y actuar en consecuencia.

- Habilidad para tomar decisiones jurídicas razonadas.

- Capacidad para redactar textos y expresarse oralmente en un lenguaje fluido y técnico, con términos jurídicos precisos y claros.

Las seis competencias de menor importancia en los cuatro grupos encuestados fueron las siguientes:

- Conocer una lengua extranjera que permita el desempeño eficiente en el ámbito jurídico (inglés, portugués o español).

- Capacidad de trabajar en equipos interdisciplinarios como experto en Derecho y contribuir de manera efectiva a sus tareas.

- Habilidad para ejercer su profesión en equipo con colegas.

- Considerar la pertinencia del uso de medios alternativos en la solución de conflictos.

- Capacidad para analizar una amplia diversidad de trabajos complejos en relación con el Derecho y sintetizar sus argumentos de forma precisa.

- Habilidad para aplicar criterios de investigación científica en su actividad profesional.

Las dos competencias más importantes para los académicos fueron:

- Capacidad de argumentar y razonar jurídicamente.

- Actuar de manera leal, diligente y transparente en la defensa de intereses de las personas a las que representa.

Para los graduados, las competencias que, a su juicio, eran más destacables fueron:

- Actuar de manera leal, diligente y transparente en la defensa de intereses de las personas a las que representa.

- Capacidad de argumentar y razonar jurídicamente. 
Reflexión de la enseñanza-aprendizaje del derecho en el siglo XXI...

Por su parte, los estudiantes establecieron como las competencias específicas más importantes:

- Capacidad de argumentar y razonar jurídicamente.

- Actuar de manera leal, diligente y transparente en la defensa de intereses de las personas a las que representa.

Para los empleadores encuestados, fueron las siguientes:

- Actuar de manera leal, diligente y transparente en la defensa de intereses de las personas a las que representa.

- Conocer, interpretar y aplicar los principios generales del Derecho y del ordenamiento jurídico.

\subsection{Implementación y puesta en práctica del modelo}

Para conseguir que al finalizar el grado de Derecho el estudiante adquiriera todas estas competencias que han sido determinadas como fundamentales, es necesario diseñar un sistema transversal. En este sentido, se explicará el sistema llevado a cabo por la Universidad de Deusto (España), cuna del Proyecto Tuning.

El Modelo de Aprendizaje de la Universidad de Deusto (MAUD) ha establecido una metodología mediante la cual estas competencias son divididas entre las distintas asignaturas de la carrera y, al mismo tiempo, en niveles que reflejan el grado de adquisición de las mismas. Cada profesor es responsable de conseguir que al finalizar el curso los estudiantes obtengan las competencias fijadas para su asignatura. Respecto a las competencias específicas, al estar relacionadas de manera directa con cada asignatura, el profesor establece las capacidades que tendrá el alumno en función de los contenidos de la misma. En los programas de las asignaturas de la Universidad de Deusto se establecen además varios niveles para competencia y, del mismo modo, indicadores que permiten conocer el grado de desarrollo. Los métodos de enseñanza para adquirirlas son distintos: en ocasiones, una determinada asignatura está más centrada en la lección expositiva, debido a que las competencias genéricas y específicas se enfocan a los conocimientos teóricos del área; en cambio, puede que para otras asignaturas estén previstas competencias de carácter más práctico como la resolución de problemas y, por tanto, sea más indicado concentrar la clase en la resolución de casos prácticos o en el análisis de la jurisprudencia.

NOVUM JUS • ISSN: 1692-6013 • Volumen 9 No. 1 • Enero - Junio 2015 • Págs. 81-102 


\subsection{Evaluación basada en competencias}

En este punto es necesario reflexionar en torno a los sistemas de evaluación que deben acompañar los cambios metodológicos explicados, como consecuencia de la convergencia global en materia de educación superior. ${ }^{29}$ La evaluación es entendida como el conjunto de actuaciones tendientes a otorgar un juicio o una valoración sobre algo con base en diversos criterios. ${ }^{30}$ La búsqueda del método más adecuado para evaluar al estudiante es uno de los puntos fundamentales, porque es la única forma veraz de conocer el grado de adquisición de las competencias genéricas y específicas asignadas al curso.

Sin lugar a dudas, tal evaluación es uno de los temas más cruciales de este modelo de enseñanza. Su dificultad reside en lograr un sistema de evaluación objetivo que dé cuenta de que las competencias se adquirieron a lo largo del curso y gracias a la metodología empleada por el docente y demostrar que el estudiante no las poseía al inicio del curso. Tyler, máximo exponente del aprendizaje basado en objetivos, señala que para obtener instrumentos de medición precisos es necesario, en primer lugar, definir los objetivos en términos de comportamiento y, en segundo lugar, diseñar métodos de evaluación que permitan a los estudiantes demostrar la consecución de dichos objetivos. ${ }^{31}$

Con base en lo anterior, se considera que lo más pertinente en la evaluación basada en competencias, una vez se cuente con un sistema de medición objetivo, es poner en práctica ese instrumento al inicio y al final del curso. De este modo podría comprobarse el grado de adquisición objetivo de determinadas competencias por parte del estudiante.

Con anterioridad al establecimiento del EEES, la enseñanza-aprendizaje del Derecho giraba, en muchos casos, en torno a la lección expositiva en la cual el profesor era el centro de atención y los estudiantes se limitaban a tomar notas de

29 "La enseñanza universitaria, en general, ha visto convertido el examen en su objetivo y fin primordial. Quizá el hallazgo de una alternativa válida al actual examen memorístico fuera un factor decisivo para la consolidación de una reforma global de los actuales esquemas docentes, en consecuencia con un entorno social en constante evolución". Antonio Francisco Fernández Tomás y Rosario Huesa Vinaixa, "La enseñanza del Derecho Internacional Público: el punto de vista del alumnado", Anales del Centro de Alzira de la Universidad Nacional de Educación a Distancia, núm. 3 (1982-1983): 29

30 Eva María Rubio, María Magnolia Pardo y Mercedes Farías, Buenas prácticas para la docencia del Derecho adaptada al EEES (Murcia: Ediciones de la Universidad de Murcia, 2010), 201.

31 Ralph Tyler, Principios básicos del currículo (Barcelona: Troquel, 1973), 20. 
Reflexión de la enseñanza-aprendizaje del derecho en el siglo XXI...

aquello que este explicaba. Debido a ello, la evaluación se reducía a un examen al final del curso en el que el estudiante plasmaba los conocimientos memorizados en los últimos días o, con suerte, en las últimas semanas. En ocasiones, se tenían en cuenta actividades prácticas desarrolladas por el alumno con anterioridad al examen, pero estas eran más bien escasas, ${ }^{32}$ es decir, la evaluación tendía a considerar como global la puntuación que se había obtenido en un único examen final. En la actualidad, con estas reformas educativas, se pretende potenciar una evaluación continua, que además esté alineada con la metodología empleada por el profesor y muestre el grado de adquisición de las competencias propuestas para el curso concreto. ${ }^{33}$

Existen distintos métodos para la evaluación de competencias, pero en este caso se seguirá el utilizado por la Universidad de Deusto y el Proyecto Tuning, porque se considera el más efectivo y no es otro que el establecido por Aurelio Villa y Manuel Poblete, máximos exponentes del modelo de enseñanza basado en la adquisición de competencias. Consiste en el establecimiento de distintos niveles de dominio, indicadores y descriptores para evaluar el grado de dicha adquisición por parte del estudiante. Así, en cuanto a la competencia genérica de pensamiento sistémico, se han definido tres áreas de dominio: organizar e integrar mentalmente diversos componentes de la realidad y explicarla con modelos holísticos; afrontar la realidad al utilizar el conocimiento con un enfoque globalizador en situaciones y tareas complejas; impactar de modo positivo en el equipo con una visión sistémica y dinámica e integrar perspectivas diversas. De la misma forma, han sido recogidos los indicadores y descriptores de los mismos, de cara a la correcta evaluación de esta competencia genérica, que se recogen en la siguiente tabla:

32 "[...] la evaluación de los estudiantes ha de prescindir, progresivamente, del método de los exámenes repetitivos de diferentes materias que concede una excesiva importancia a la memoria o al amontonamiento temporal de los conocimientos y que no ofrece ninguna garantía ni ninguna indicación de la capacidad de los estudiantes para afrontar la futura actividad profesional. Por el contrario, los exámenes deberían consistir en pruebas en que los estudiantes, con su propio material, fueran capaces de dar cuenta de su comprensión de la materia y sobre su modo de reflexionar y abordar las cuestiones a la que se les someten". Bricall, "Informe Universidad 2000", http://www.observatoriuniversitari.org/es/files/2014/05/Bricall_JM-2000-Informe-Universidad-2000.pdf (acceso, julio 14, 2014).

33 Daniel García San José, La enseñanza-aprendizaje del Derecho Internacional Público en el Espacio Europeo de Educación Superior (Murcia: Ediciones Laborum, 2005), 72.

NOVUM JUS • ISSN: 1692-6013 • Volumen 9 Nº. 1 • Enero - Junio 2015 • Págs. 81-102 
Tabla 1

Evaluación de la competencia de pensamiento sistémico

\begin{tabular}{|c|c|c|c|c|c|c|}
\hline \multirow{2}{*}{ Niveles de dominio } & \multirow{2}{*}{ Indicadores } & \multicolumn{5}{|c|}{ Descriptores } \\
\hline & & 1 & 2 & 3 & 4 & 5 \\
\hline \multirow{4}{*}{$\begin{array}{l}\text { Primer nivel de } \\
\text { dominio: organizar e } \\
\text { integrar mentalmente } \\
\text { diversos componen- } \\
\text { tes de la realidad y } \\
\text { explicarla mediante } \\
\text { modelos holísticos }\end{array}$} & $\begin{array}{l}\text { Integra distintos } \\
\text { elementos de la asig- } \\
\text { natura en su análisis. }\end{array}$ & $\begin{array}{l}\text { No relaciona ele- } \\
\text { mentos fundamen- } \\
\text { tales de la asignatura } \\
\text { o hace relaciones } \\
\text { incoherentes. }\end{array}$ & $\begin{array}{c}\text { Establece relaciones } \\
\text { significativas entre } \\
\text { algunos elementos, } \\
\text { hechos, etc. de la } \\
\text { asignatura. }\end{array}$ & $\begin{array}{l}\text { Explica procesos, } \\
\text { relaciones, interac- } \\
\text { ciones, conexiones, } \\
\text { dependencias, etc. de } \\
\text { la asignatura. }\end{array}$ & $\begin{array}{l}\text { Ordena y jerarquiza } \\
\text { elementos, hechos, } \\
\text { procesos, relaciones, } \\
\text { etc. de la asignatura. }\end{array}$ & $\begin{array}{l}\text { Integra los procesos, } \\
\text { las relaciones, interac- } \\
\text { ciones, etc. de la asig- } \\
\text { natura en un esquema } \\
\text { explicativo. }\end{array}$ \\
\hline & $\begin{array}{c}\text { Demuestra capacidad } \\
\text { para transferir los co- } \\
\text { nocimientos teóricos o } \\
\text { del aula a las situacio- } \\
\text { nes prácticas. }\end{array}$ & $\begin{array}{c}\text { No relaciona teoría y } \\
\text { práctica. }\end{array}$ & $\begin{array}{c}\text { Reflexiona con } \\
\text { dificultad sobre las } \\
\text { aplicaciones prácti- } \\
\text { cas de lo aprendido. }\end{array}$ & $\begin{array}{l}\text { Identifica aplicaciones } \\
\text { prácticas de los conte- } \\
\text { nidos estudiados. }\end{array}$ & $\begin{array}{c}\text { Realiza algunas aplica- } \\
\text { ciones prácticas de los } \\
\text { contenidos. }\end{array}$ & $\begin{array}{c}\text { Proyecta y aplica } \\
\text { sistemáticamente los } \\
\text { contenidos sobre la } \\
\text { práctica. }\end{array}$ \\
\hline & $\begin{array}{l}\text { Considera los modelos } \\
\text { mentales que subya- } \\
\text { cen en la situación. }\end{array}$ & $\begin{array}{l}\text { Es ajeno a la exis- } \\
\text { tencia de modelos } \\
\text { mentales en la } \\
\text { situación. }\end{array}$ & $\begin{array}{c}\text { Identifica con } \\
\text { dificultades algunos } \\
\text { modelos mentales } \\
\text { que subyacen en la } \\
\text { situación. }\end{array}$ & $\begin{array}{c}\text { Reflexiona sobre el } \\
\text { impacto de los mode- } \\
\text { los mentales sobre la } \\
\text { situación. }\end{array}$ & $\begin{array}{l}\text { Identifica y explica } \\
\text { los modelos mentales } \\
\text { que subyacen en la } \\
\text { situación. }\end{array}$ & $\begin{array}{c}\text { Compara y contrasta } \\
\text { distintos modelos men- } \\
\text { tales que subyacen en } \\
\text { la situación. }\end{array}$ \\
\hline & $\begin{array}{l}\text { Utiliza algunas téc- } \\
\text { nicas básicas para el } \\
\text { desarrollo del pensa- } \\
\text { miento sistémico. }\end{array}$ & $\begin{array}{l}\text { Solo identifica } \\
\text { pares aislados de } \\
\text { causa-efecto. }\end{array}$ & $\begin{array}{c}\text { Se limita a hacer } \\
\text { análisis lineales } \\
\text { de secuencias de } \\
\text { hechos. }\end{array}$ & $\begin{array}{l}\text { Hace un análisis cir- } \\
\text { cular de las secuencias } \\
\text { de hechos. }\end{array}$ & $\begin{array}{c}\text { Capta las interacciones } \\
\text { circulares con acierto } \\
\text { y agilidad. }\end{array}$ & $\begin{array}{l}\text { Identifica los efectos } \\
\text { de la intervención y } \\
\text { la regulación en las } \\
\text { secuencias circulares. }\end{array}$ \\
\hline
\end{tabular}




\begin{tabular}{|c|c|c|c|c|c|c|}
\hline \multirow{2}{*}{ Niveles de dominio } & \multirow{2}{*}{ Indicadores } & \multicolumn{5}{|c|}{ Descriptores } \\
\hline & & 1 & 2 & 3 & 4 & 5 \\
\hline \multirow{6}{*}{$\begin{array}{l}\text { Segundo nivel de } \\
\text { dominio: } \\
\text { Afrontar la realidad } \\
\text { utilizando el cono- } \\
\text { cimiento con un } \\
\text { enfoque globalizador } \\
\text { en situaciones y tareas } \\
\text { complejas. }\end{array}$} & $\begin{array}{c}\text { Integra elementos de } \\
\text { distintas asignaturas o } \\
\text { áreas en su análisis de } \\
\text { realidad. }\end{array}$ & $\begin{array}{l}\text { No relaciona ele- } \\
\text { mentos diferentes de } \\
\text { asignaturas. }\end{array}$ & $\begin{array}{l}\text { Establece relaciones } \\
\text { entre algunos } \\
\text { elementos, hechos, } \\
\text { etc. de diferentes } \\
\text { asignaturas. }\end{array}$ & $\begin{array}{c}\text { Integra elementos } \\
\text { de varias asignaturas } \\
\text { de la misma área de } \\
\text { conocimiento en un } \\
\text { modelo de análisis de } \\
\text { la realidad. }\end{array}$ & $\begin{array}{l}\text { Integra elementos de } \\
\text { distintas disciplinas o } \\
\text { áreas del conocimien- } \\
\text { to en un modelo de } \\
\text { análisis de la realidad. }\end{array}$ & $\begin{array}{l}\text { Trabaja con un enfoque } \\
\text { interdisciplinar. }\end{array}$ \\
\hline & $\begin{array}{l}\text { Recurre a diversas } \\
\text { perspectivas, fuentes, } \\
\text { dimensiones, etc. para } \\
\text { analizar la realidad. }\end{array}$ & $\begin{array}{l}\text { Recurre a una única } \\
\text { perspectiva, fuente, } \\
\text { dimensión, etc. en } \\
\text { su análisis de la } \\
\text { realidad. }\end{array}$ & $\begin{array}{l}\text { Recurre a pocas } \\
\text { perspectivas, fuen- } \\
\text { tes, dimensiones, } \\
\text { etc. en su análisis de } \\
\text { la realidad. }\end{array}$ & $\begin{array}{l}\text { Recurre a una variedad } \\
\text { de perspectivas, fuen- } \\
\text { tes, dimensiones, etc. } \\
\text { para poder compren- } \\
\text { der la realidad. }\end{array}$ & $\begin{array}{c}\text { Ordena y priori- } \\
\text { za entre diversas } \\
\text { perspectivas, fuentes, } \\
\text { dimensiones, etc. } \\
\text { en su análisis de la } \\
\text { realidad. }\end{array}$ & $\begin{array}{c}\text { Integra con cohe- } \\
\text { rencia las diversas } \\
\text { perspectivas, fuentes, } \\
\text { dimensiones, etc. que } \\
\text { utiliza en su análisis de } \\
\text { la realidad. }\end{array}$ \\
\hline & $\begin{array}{l}\text { Transfiere los con- } \\
\text { tenidos a la práctica } \\
\text { para integrarlos en un } \\
\text { proyecto. }\end{array}$ & $\begin{array}{l}\text { No aplica los } \\
\text { contenidos sobre la } \\
\text { práctica. }\end{array}$ & $\begin{array}{c}\text { Hace aplicaciones } \\
\text { parciales de los } \\
\text { contenidos a la } \\
\text { práctica. }\end{array}$ & $\begin{array}{l}\text { Aplica los contenidos a } \\
\text { la práctica y los integra } \\
\text { en un proyecto. }\end{array}$ & $\begin{array}{l}\text { Diseña y desarrolla } \\
\text { un proyecto en el que } \\
\text { aplica los contenidos. }\end{array}$ & $\begin{array}{l}\text { Evalúa su proyecto } \\
\text { y adopta mejoras en } \\
\text { consecuencia. }\end{array}$ \\
\hline & $\begin{array}{l}\text { Identifica y explica los } \\
\text { macrosistemas rele- } \\
\text { vantes en la situación. }\end{array}$ & $\begin{array}{c}\text { No diferencia el } \\
\text { macrosistema de } \\
\text { los niveles meso y } \\
\text { micro. }\end{array}$ & $\begin{array}{l}\text { Solo describe los } \\
\text { macrosistemas en la } \\
\text { situación. }\end{array}$ & $\begin{array}{l}\text { Explica los macrosis- } \\
\text { temas relevantes en la } \\
\text { situación. }\end{array}$ & $\begin{array}{c}\text { Establece relaciones } \\
\text { entre niveles macro, } \\
\text { meso y micro. }\end{array}$ & $\begin{array}{c}\text { Es capaz de presentar } \\
\text { con claridad las inte- } \\
\text { rrelaciones entre los } \\
\text { niveles macro, meso y } \\
\text { micro. }\end{array}$ \\
\hline & $\begin{array}{l}\text { Toma conciencia de } \\
\text { sus propios modelos } \\
\text { mentales. }\end{array}$ & $\begin{array}{c}\text { No es consciente de } \\
\text { sus propios modelos } \\
\text { mentales. }\end{array}$ & $\begin{array}{l}\text { Tiene dificultades } \\
\text { para reconocer sus } \\
\text { propios modelos } \\
\text { mentales. }\end{array}$ & $\begin{array}{l}\text { Se muestra consciente } \\
\text { de sus propios mode- } \\
\text { los mentales. }\end{array}$ & $\begin{array}{c}\text { Considera otros mode- } \\
\text { los mentales diferentes } \\
\text { al suyo. }\end{array}$ & $\begin{array}{l}\text { Explica la situación } \\
\text { desde modelos menta- } \\
\text { les diferentes al suyo. }\end{array}$ \\
\hline & $\begin{array}{l}\text { Diferencia entre el } \\
\text { dato aislado y las } \\
\text { generalizaciones infe- } \\
\text { ridas de los datos. }\end{array}$ & $\begin{array}{l}\text { Generaliza impul- } \\
\text { sivamente y sin } \\
\text { ninguna precaución. }\end{array}$ & $\begin{array}{l}\text { Confunde el dato } \\
\text { aislado y la gene- } \\
\text { ralización, aunque } \\
\text { muestra cierta } \\
\text { precaución. }\end{array}$ & $\begin{array}{c}\text { Generaliza con } \\
\text { precaución y sentido } \\
\text { crítico a partir de las } \\
\text { observaciones. }\end{array}$ & $\begin{array}{l}\text { Utiliza las generaliza- } \\
\text { ciones en la toma de } \\
\text { decisiones de acuerdo } \\
\text { con su grado de } \\
\text { precisión. }\end{array}$ & $\begin{array}{l}\text { Contrasta la sistemáti- } \\
\text { camente la validez de } \\
\text { las generalizaciones. }\end{array}$ \\
\hline
\end{tabular}

Fuente: Aurelio Villa Sánchez y Manuel Poblete Ruiz, Aprendizaje basado en competencias. Una propuesta para la evaluación de las competencias genéricas (Bilbao: Universidad de Deusto, 2007), 73-75. 


\section{Conclusiones}

A la luz de las transformaciones producidas por la internacionalización de la sociedad actual, se hacía necesaria una reforma de los modelos de enseñanza-aprendizaje, tendiente a la formación de ciudadanos en este nuevo contexto; por lo tanto, se encuentra justificada. En el seno de la Unión Europea, la excelencia educativa y la armonización de los sistemas y títulos de enseñanza superior son un instrumento clave en la consecución del mercado interior europeo, en cuanto a movilidad de trabajadores y estudiantes. Por otra parte, se ha evidenciado que la convergencia educativa superior ha adquirido una importancia global, ya que se ideó en el continente europeo y hoy en día estas reformas educativas se han difundido por el mundo. Los objetivos marcados por la convergencia global en materia educativa no pueden quedarse en una simple "redenominación" de las titulaciones y asignaturas, sino que debe suponer una verdadera transformación de la manera en la que es concebida la educación universitaria en términos de métodos, objetivos y evaluación. Es preciso y urgente analizar la calidad y pertinencia de los métodos que se están utilizando en la enseñanza-aprendizaje del Derecho y, en caso de que estos no correspondan a los estándares de calidad y excelencia perseguidos, será necesario buscar nuevos modelos más acordes con estas y con los requerimientos de la sociedad contemporánea.

La enseñanza basada en competencias ha constituido el modelo más idóneo para llevar a cabo las transformaciones educativas demandadas por la sociedad del siglo XXI. Su éxito radica en centrar la enseñanza en el estudiante y en su adquisición de competencias genéricas y específicas a lo largo del curso, afines a la sociedad actual. Sin embargo, la consecución de este enfoque requiere un esfuerzo sin referente por parte de las instituciones de educación superior, tanto en términos materiales como personales. Asimismo, hay que tener en cuenta sus falencias, sobre todo en términos de evaluación, para crear instrumentos de evaluación objetivos que posibiliten una verdadera alineación entre objetivos, metodología y evaluación.

\section{Referencias}

Aneca. "Libro Blanco. Título de grado en Derecho". http://www.aneca.es/var/media/150240/ libroblanco_derecho_def.pdf (acceso marzo 1, 2013).

Barnett, Ronald. Los límites de la competencia. El conocimiento, la educación superior y la sociedad. Barcelona: Gedisa, 2001. 
Reflexión de la enseñanza-aprendizaje del derecho en el siglo XXI...

Beck, Ulrich. ¿Qué es la globalización? Falacias del globalismo, respuestas a la globalización. Barcelona: Paidós, 1998.

Bricall, Josep M. "Informe Universidad 2000". http://www.observatoriuniversitari.org/es/ files/2014/05/Bricall_JM-2000-Informe-Universidad-2000.pdf (acceso, julio 14, 2014).

Comisión Europea. "New Skills for New Jobs". http://ec.europa.eu/social/main.jsp?catId=568 (acceso marzo 1, 2013).

De Julios Campuzano, Alfonso. La transición paradigmática de la teoría jurídica. El Derecho ante la globalización. Madrid: Dykinson, 2009.

Fernández Tomás, Antonio Francisco y Rosario Huesa Vinaixa. "La enseñanza del Derecho Internacional Público: el punto de vista del alumnado". Anales del Centro de Alzira de la Universidad Nacional de Educación a Distancia, núm. 3 (1982-1983): 17-32.

García Fraile, Juan Antonio y Carmen Saban Vera. Un nuevo modelo de formación para el siglo XXI: la enseñanza basada en competencias. Barcelona: Davinci, 2008.

García San José, Daniel. La enseñanza-aprendizaje del Derecho Internacional Público en el Espacio Europeo de Educación Superior. Murcia: Ediciones Laborum, 2005.

González, Julia y Robert Wagennar. Tuning Educational Structures in Europe. La contribución de las universidades al proceso de Bolonia. Una introducción. Bilbao: Universidad de Deusto, 2006.

Hipkins, Rosemary. The Nature of the Key Competences. A Background Paper. Nueva Zelanda: Wellignton, 2006.

Jiménez Eguizábal, Alfredo (dir.). Proyecto piloto de adaptación de asignaturas de Pedagogía y Psicología al sistema de créditos europeos ECTS. Burgos: Universidad de Burgos, 2005.

Litvinoff, Saul. "Derecho Global, Derecho Civil y Common Law". Foro de Derecho Mercantil: revista internacional, núm. 3 (2004): 99-108.

Magna Charta Universitatum. Bolonia, 18 de septiembre de 1988.

Ministros europeos de Educación Superior. Declaración de Bolonia. Bolonia, 19 de junio de 1999.

Ministros europeos de Educación Superior. Declaración de La Sorbona. París, 25 de mayo de 1998.

Morales Vallejo, Pedro. "Implicaciones para el profesor de una enseñanza centrada en el alumno". Miscelánea Comillas 64, núm. 124 (2006): 11-38.

OCDE. "Definition and Selection of Competencies: Theoretical and Conceptual Foundations". http://www.oecd.org/edu/skills-beyond-school/41529556.pdf (acceso marzo 1, 2013).

Ortega y Gasset, José. Misión de la universidad. Buenos Aires: Edición de Palma, 2001.

Rodríguez-Arana, Jaime y Rafael Palomino Lozano (dirs.). Enseñar Derecho en el siglo XXI. Una guía práctica sobre el grado en Derecho. Pamplona: Thomson Aranzadi, 2009.

Rubio, Eva María, María Magnolia Pardo y Mercedes Farías. Buenas prácticas para la docencia del Derecho adaptada al EEES. Murcia: Ediciones de la Universidad de Murcia, 2010.

NOVUM JUS • ISSN: 1692-6013 • Volumen 9 No .1 • Enero - Junio 2015 • Págs. 81-102 
Sáenz de Miera, Antonio. La universidad en la nueva economía. Madrid: Secretaría General Técnica, 2002.

Santos Pastor, Marisa, Luis Fernando Martínez Muñoz y Víctor Manuel López Pastor. La innovación docente en el EEES. Almería: Universidad de Almería, 2009.

Scheurman, William. "Reflexive Law and the Challenge of Globalization". Journal of Political Philosophy 9, núm. 1 (2001): 81-102.

Tyler, Ralph. Principios básicos del currículo. Barcelona: Troquel, 1973.

Unesco. "Declaración mundial sobre la educación superior del siglo XXI". http://www. unesco.org/education/educprog/wche/declaration_spa.htm (acceso marzo 1, 2013).

Villa, Aurelio y Manuel Poblete. Aprendizaje basado en competencias. Una propuesta para la evaluación de las competencias genéricas. Bilbao: Universidad de Deusto, 2007.

Viso Alonso, José Ramiro. Qué son las competencias. Enseñar y aprender por competencias. Madrid: EOS, 2010.

XXII Congreso Mundial de Filosofía Jurídica y Social. "Declaración de Granada sobre la globalización". http://www.globalizacion.org/globalizacion/GlbzDeclaracionGranada. htm (acceso febrero 4, 2013).

Zumbansen, Peer. "Transnational Law" en Elgar Encyclopedia of Comparative Law. Londres: Edward Elgar Publishing Ltd, 2006. 